\title{
LINEAR FUNCTIONALS CONNECTED WITH STRONG DOUBLE CESARO SUMMABILITY
}

\author{
FATIH NURAY ${ }^{1}$ AND NIMET PANCAROḠLU AKIN ${ }^{2, *}$ \\ ${ }^{1}$ Department of Mathematics, Afyon Kocatepe University, Turkey \\ ${ }^{2}$ Department of Mathematics and Science Education, Afyon Kocatepe University, Turkey \\ *Corresponding author: npancaroglu@aku.edu.tr
}

\begin{abstract}
D. Borwein characterized linear functionals on the normed linear spaces $w_{p}$ and $W_{p}$. In this paper we extend his results by presenting definitions for the double strong Cesaro mean. Using these new notions of strongly $p$-Cesaro summable double sequence and strongly $p$-Cesaro summable bivariate function we present extensions of D. Borwein's results.
\end{abstract}

\section{INTRODUCTION}

The first definitions and investigations of the convergence of double sequences are usually atributted to F. Pringsheim [12], who studied such sequences and series more than hundred years ago. Pringsheim defined what we call the $\mathrm{P}$ limit and gave examples of convergence (P convergence) of double sequences with and without the usual convergence of rows and columns. G. H. Hardy [4], considered in more details the case of convergence of double sequences where, besides the existence of the $\mathrm{P}$ limit, rows and columns converge. F. Moricz [6-8] discovered an alternative approach to the Hardy convergence, which significantly influenced the whole theory.

The following notion of convergence for double sequences was presented by Pringsheim in [11]. A double sequence $x=\left\{x_{n m}\right\}$ of real numbers is said to be convergent to $L \in \mathbb{R}$ in Pringsheim's sense if for any $\varepsilon>0$,

Received 2019-01-10; accepted 2019-02-12; published 2019-05-01.

2010 Mathematics Subject Classification. Primary 40A05; Secondary 40C05.

Key words and phrases. double sequence; measurable function; bivariate function; Cesaro summable function.

(c)2019 Authors retain the copyrights of their papers, and all open access articles are distributed under the terms of the Creative Commons Attribution License. 
there exists $N_{\varepsilon} \in \mathbb{N}$ such that $\left|x_{n m}-L\right|<\varepsilon$, whenever $n, m>N_{\varepsilon}$. In this case we denote such limit as follow:

$$
P-\lim _{n, m \rightarrow \infty} x_{n m}=L .
$$

A classical notion of sequence space is the following:

$$
w_{p}=\left\{x=\left(x_{n}\right): \lim _{N \rightarrow \infty} \frac{1}{N} \sum_{n=1}^{N}\left|x_{n}-\ell\right|^{p}=0\right\} .
$$

In [2], D. Borwein extended the sequence space $w_{p}$ to the function space $W_{p}$, the space of real valued functions $x$, measurable (in the Lebesque sense) in the interval $(1, \infty)$ for which there is a number $\ell=\ell_{x}$ such that

$$
\lim _{T \rightarrow \infty} \frac{1}{T} \int_{1}^{T}|x(t)-\ell|^{p}=0
$$

By a linear functional we mean one that is real-valued, additive, homogeneous and continuous. It is to be supposed throughout that $1 \leq p<\infty$ and that $\frac{1}{p}+\frac{1}{q}=1$.

\section{Main Results}

We begin to the main results with following definitions:

Definition 2.1. Let $x=\left\{x_{n m}\right\}$ be a real double sequence. Then the double sequence $x$ is said to be strongly p-Cesaro summable to $\ell$ if

$$
P-\lim _{N, M \rightarrow \infty} \frac{1}{N M} \sum_{n=1}^{N} \sum_{m=1}^{M}\left|x_{n m}-\ell\right|^{p}=0 .
$$

The space of all strongly $p$-Cesaro summable double sequences will be denote by $w_{p}^{2}$. Observe that this space is normed by

$$
\|x\|_{2}=\sup _{N, M \geq 1}\left(\frac{1}{N M} \sum_{n=1}^{N} \sum_{m=1}^{M}\left|x_{n m}-\ell\right|^{p}\right)^{\frac{1}{p}} .
$$

Definition 2.2. Let $x$ be a real valued bivariate function, measurable (in the Lebesque sense) in the $(1, \infty) \times$ $(1, \infty)$. Then the bivariate function $x$ is said to be strongly $p$-Cesaro summable to $\ell$ if

$$
\lim _{T, R \rightarrow \infty} \frac{1}{T R} \int_{1}^{T} \int_{1}^{R}|x(t, r)-\ell|^{p} d r d t=0 .
$$

The space of all strongly $p$-Cesaro summable bivariate functions will be denote by $W_{p}^{2}$. Observe that this space is normed by

$$
\|x\|_{2}=\sup _{T \geq 1, R \geq 1}\left(\frac{1}{T R} \int_{1}^{T} \int_{1}^{R}|x(t, r)-\ell|^{p} d r d t\right)^{\frac{1}{p}} .
$$

Given any real double sequence $\alpha=\left\{\alpha_{n m}\right\}$. We define a double sequence $\left\{m_{n m}(\alpha, p)\right\}$ by 


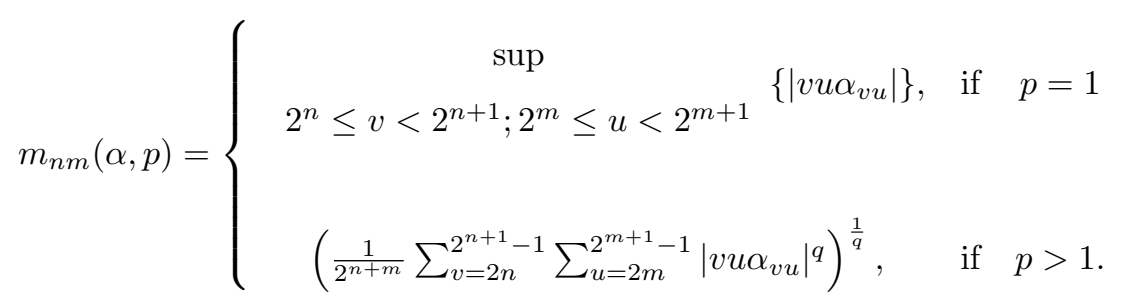

Given any real real valued bivariate function $\alpha(t, r)$ measurable in $(1, \infty) \times(1, \infty)$. We define a double sequence $\left\{M_{n m}(\alpha, p)\right\}$ by

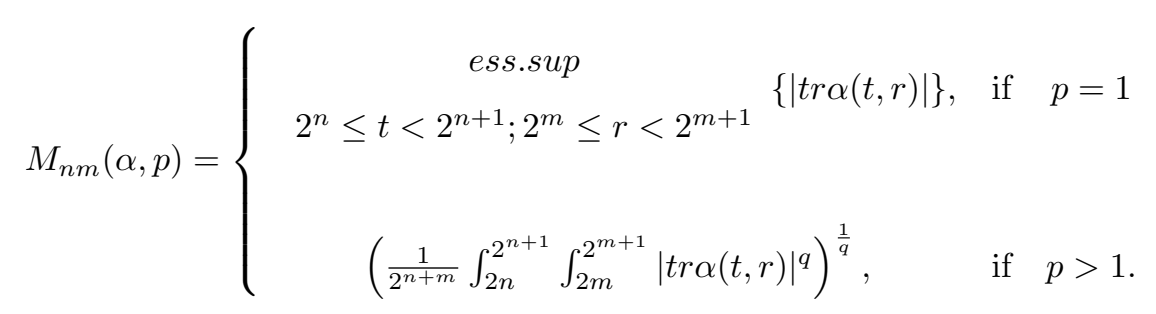

Theorem 2.1. $\quad$ (i) If $f$ is a linear functional on $W_{p}^{2}$, then there is a real number a and a real valued bivariate function $\alpha$, measurable in $(1, \infty) \times(1, \infty)$ such that

$$
f(x)=a \ell+\int_{1}^{\infty} \int_{1}^{\infty} \alpha(t, r) x(t, r) d r d t
$$

for every $x \in W_{p}^{2}$ and

$$
\sum_{n=0}^{\infty} \sum_{m=0}^{\infty} M_{n m}(\alpha, p)<\infty .
$$

(ii) If $a$ is a real number and $\alpha$ is a real valued bivariate function, measurable in $(1, \infty) \times(1, \infty)$, satisfying (2.2), then (2.1) defines a linear function on $W_{p}^{2}$ with

$$
\|f\|_{2} \leq|a|+2^{\frac{2}{p}} \sum_{n=0}^{\infty} \sum_{m=0}^{\infty} M_{n m}(\alpha, p)
$$

and the integral in (2.1) is absolutely convergent for every $x \in W_{p}^{2}$.

Proof. Let $L_{p}^{2}$ be the linear space of real valued bivariate functions $x$ measurable in $(1, \infty) \times(1, \infty)$ for which

$$
\int_{1}^{\infty} \int_{1}^{\infty}|x(t, r)|^{p} d r d t<\infty
$$

with norm

$$
\|x\|_{L_{p}^{2}}=\left(\int_{1}^{\infty} \int_{1}^{\infty}|x(t, r)|^{p} d r d t\right)^{\frac{1}{p}} .
$$

Clearly, if $x \in L_{p}^{2}$, then $x \in W_{p}^{2}, \ell=0$ and $\|x\|_{2}=\|x\|_{W_{p}^{2}} \leq\|x\|_{L_{p}^{2}}$. Consequently the restriction to $L_{p}^{2}$ of the given linear functional $f$ on $W_{p}^{2}$ is linear on $L_{p}^{2}$. It follows from standard results that there is a real valued bivariate function $\alpha$, measurable in $(1, \infty) \times(1, \infty)$, such that

$$
f(x)=\int_{1}^{\infty} \int_{1}^{\infty} \alpha(t, r) x(t, r) d r d t
$$


for all $x \in L_{p}^{2}$ and either

$$
\begin{aligned}
& \text { ess.sup }\{|\alpha(t, r)|\}<\infty \quad \text { if } p=1 \\
& 1 \leq t<\infty \\
& 1 \leq r<\infty
\end{aligned}
$$

or

$$
\int_{1}^{\infty} \int_{1}^{\infty}|\alpha(t, r)|^{q} d r d t<\infty \quad \text { if } p>1
$$

To show that $\alpha$ must necessarily satisfy (2.2) we consider the cases $p=1$ and $p>1$ separately. If $p=1$, let $M_{n m}=M_{n m}(\alpha, 1)$. There is a measurable set $e_{n m}$ of positive measure $\left|e_{n m}\right|$ in the $\left(2^{n}, 2^{n+1}\right) \times\left(2^{m}, 2^{m+1}\right)$ such that

$$
|\operatorname{tr} \alpha(t, r)|>M_{n m}-\frac{1}{2^{n+m}}
$$

for all $(t, r) \in e_{n m}$.

Let

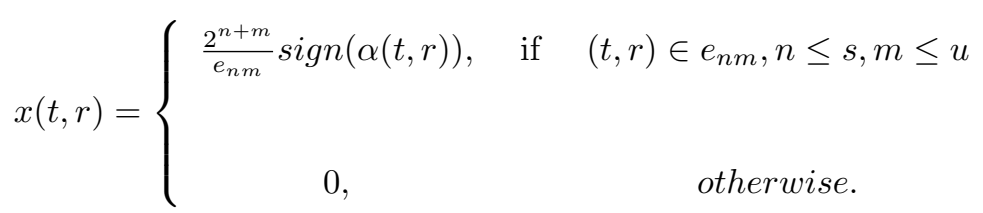

Then $x \in L_{1}^{2}$ and so, by (2.3),

$$
\begin{aligned}
\|f\|_{2}\|x\|_{2} \geq f(x)= & \int_{1}^{\infty} \int_{1}^{\infty} x(t, r) \alpha(t, r) d r d t \\
& =\sum_{n=0}^{s} \sum_{m=0}^{u} \iint_{e_{n m}} \frac{2^{n+m}}{\left|e_{n m}\right|}|\alpha(t, r)| d r d t \\
& \geq \frac{1}{4} \sum_{n=0}^{s} \sum_{m=0}^{u} \frac{1}{\left|e_{n m}\right|} \iint_{e_{n m}}|t r \alpha(t, r)| d r d t \\
& \geq \frac{1}{4} \sum_{n=0}^{s} \sum_{m=0}^{u}\left(M_{n m}-\frac{1}{2^{n+m}}\right) .
\end{aligned}
$$

Furtermore, for $2^{z} \leq T<2^{z+1} \leq 2^{s+1}, 2^{h} \leq R<2^{h+1} \leq 2^{u+1}$,

$$
\begin{aligned}
\frac{1}{T R} \int_{1}^{T} \int_{1}^{R}|x(t, r)| d r d t & \leq \frac{1}{2^{z+h}} \int_{1}^{2^{z+1}} \int_{1}^{2^{h+1}}|x(t, r)| d r d t \\
& =\frac{1}{2^{z+h}} \sum_{n=0}^{z} \sum_{m=0}^{h} \iint_{e n m} x(t, r) \mid d r d t \\
& \leq \frac{1}{2^{z+h}} \sum_{n=0}^{z} \sum_{m=0}^{h} 2^{n+m}<4,
\end{aligned}
$$

and for $T>2^{s+1}, R>2^{u+1}$

$$
\frac{1}{T R} \int_{1}^{T} \int_{1}^{R}|x(t, r)| d r d t \leq \frac{1}{2^{s+1} 2^{u+1}} \int_{1}^{2^{s+1}} \int_{1}^{2^{u+1}}|x(t, r)| d r d t<1 .
$$


Hence $\|x\|_{2}<4$ and so, by (2.4),

$$
4\|f\|_{2}+\frac{1}{4} \sum_{n=0}^{\infty} \sum_{m=0}^{\infty} \frac{1}{2^{n+m}}=4\|f\|_{2}+1 \geq \frac{1}{4} \sum_{n=0}^{\infty} \sum_{m=0}^{\infty} M_{n m},
$$

which establishes (2.2) in this case. If $p>1$, let $M_{n m}=M_{n m}(\alpha, p)$ and let

$$
x(t, r)=\left\{\begin{array}{ccc}
\frac{(t r)^{q}}{2^{n+m}}\left|\frac{\alpha(t, r)}{M_{n m}}\right|^{\frac{q}{p}} \operatorname{sign}(\alpha(t, r)), & \text { if } & 2^{n} \leq t<2^{n+1} \leq 2^{z+1} ; \\
& 2^{m} \leq r<2^{m+1} \leq 2^{u+1} \text { and } M_{n m} \neq 0 \\
0, & \text { otherwise. }
\end{array}\right.
$$

Then $x \in L_{p}^{2}$ and so, by (2.3),

$$
\begin{gathered}
f(x)=\int_{1}^{2^{z+1}} \int_{1}^{2^{u+1}}|\alpha(t, r) x(t, r)| d r d t=\sum_{n=0}^{z} \sum_{m=0}^{u} \int_{2^{n}}^{2^{n+1}} \int_{2^{m}}^{2^{m+1}}|\alpha(t, r) x(t, r)| d r d t \\
=\sum_{n=0}^{z} \sum_{m=0}^{u} M_{n m} .
\end{gathered}
$$

Furtermore, for $2^{z} \leq T<2^{z+1} \leq 2^{s+1}, 2^{h} \leq R<2^{h+1} \leq 2^{u+1}$,

$$
\begin{aligned}
\frac{1}{T R} \int_{1}^{T} \int_{1}^{R}|x(t, r)|^{p} d r d t & \leq \frac{1}{2^{z+h}} \int_{1}^{2^{z+1}} \int_{1}^{2^{h+1}}|x(t, r)|^{p} d r d t \\
& =\frac{1}{2^{z+h}} \sum_{n=0}^{z} \sum_{m=0}^{h} \iint_{e_{n m}}|x(t, r)|^{p} d r d t \\
& \leq \frac{2^{2 p}}{2^{z+h}} \sum_{n=0}^{z} \sum_{m=0}^{h} 2^{n+m}<2^{2 p+2}
\end{aligned}
$$

and for $T>2^{z+1}, R>2^{h+1}$

$$
\frac{1}{T R} \int_{1}^{T} \int_{1}^{R}|x(t, r)|^{p} d r d t \leq \frac{1}{2^{z+1} 2^{h+1}} \int_{1}^{2^{z+1}} \int_{1}^{2^{h+1}}|x(t, r)|^{p} d r d t<4^{p} .
$$

Hence $\|x\|_{2}<2^{2+\frac{2}{p}}$ and so, by (2.5),

$$
\sum_{n=0}^{\infty} \sum_{m=0}^{\infty} M_{n m} \leq 2^{2+\frac{2}{p}}\|f\|_{2}
$$

which established (2.2) in this case.

Suppose now $p \geq 1, M_{n m}=M_{n m}(\alpha, p)$ and $x \in W_{p}^{2}$. Then by Hölder inequality

$$
\begin{aligned}
\int_{1}^{\infty} \int_{1}^{\infty} \mid \alpha(t, r) & x(t, r)\left|d r d t=\sum_{n=0}^{\infty} \sum_{m=0}^{\infty} \int_{2^{n}}^{2^{n+1}} \int_{2^{m}}^{2^{m+1}}\right| \alpha(t, r) x(t, r) \mid d r d t \\
\leq & \sum_{n=0}^{\infty} \sum_{m=0}^{\infty} M_{n m}\left(2^{p\left(1-\frac{1}{p}\right)(n+m)} \int_{2^{n}}^{2^{n+1}} \int_{2^{m}}^{2^{m+1}}\left|\frac{x(t, r)}{t r}\right|^{p} d r d t\right)^{\frac{1}{p}} \\
& \leq \sum_{n=0}^{\infty} \sum_{m=0}^{\infty} M_{n m}\left(2^{-(n+m)} \int_{2^{n}}^{2^{n+1}} \int_{2^{m}}^{2^{m+1}}|x(t, r)|^{p} d r d t\right)^{\frac{1}{p}}
\end{aligned}
$$




$$
\leq 2^{\frac{2}{p}}\|x\|_{2} \sum_{n=0}^{\infty} \sum_{m=0}^{\infty} M_{n m}
$$

It follows that

$$
\int_{1}^{\infty} \int_{1}^{\infty}|\alpha(t, r) x(t, r)| d r d t<\infty
$$

whenever $x \in W_{p}^{2}$, and in particular since the characteristic function of $(1, \infty) \times(1, \infty)$ is in $W_{p}^{2}$, that

$$
\int_{1}^{\infty} \int_{1}^{\infty}|\alpha(t, r)| d r d t<\infty
$$

Suppose next that $x \in W_{p}^{2}$ and $\ell=\ell_{x}$. Let

$$
\begin{gathered}
y(t, r)=x(t, r)-\ell \\
y_{n m}(t, r)=\left\{\begin{array}{ccc}
y(t, r), & \text { if } & 1 \leq t \leq n, 1 \leq r \leq m ; \\
0, & \text { if } & t \geq n \text { and } r \geq m .
\end{array}\right.
\end{gathered}
$$

Then $y \in W_{p}^{2}, y_{n m} \in L_{p}^{2}$ and

$$
\left\|y_{n m}-y\right\|_{2}=\sup _{T \geq n, R \geq m}\left(\frac{1}{T R} \int_{n}^{T} \int_{m}^{R}|x(t, r)-\ell|^{p}\right)^{\frac{1}{p}}=o(1) \text { as } n, m \rightarrow \infty .
$$

But

$$
\left|f\left(y_{n m}-y\right)\right|=\left|f\left(y_{n m}\right)-f(y)\right| \leq\left\|y_{n m}-y\right\|_{2}\|f\|_{2}
$$

and so, by (2.3),

$$
\begin{aligned}
f(y)= & P-\lim _{n, m \rightarrow \infty} f\left(y_{n m}\right)=P-\lim _{n, m \rightarrow \infty} \int_{1}^{n} \int_{1}^{m} y(t, r) \alpha(t, r) d r d t \\
& =\int_{1}^{\infty} \int_{1}^{\infty} x(t, r) \alpha(t, r) d r d t-\ell \int_{1}^{\infty} \int_{1}^{\infty} \alpha(t, r) d r d t .
\end{aligned}
$$

Since both integrals on the right hand side have been shown to be absolutely convergent. Taking $\delta$ to be characteristic function of $(1, \infty) \times(1, \infty)$ we see that

$$
f(x)=f(y+\ell \delta) f(y)+\ell f(\delta)=\int_{1}^{\infty} \int_{1}^{\infty} x(t, r) \alpha(t, r) d r d t+a \ell
$$

where $a=f(\delta)-\int_{1}^{\infty} \int_{1}^{\infty} \alpha(t, r)$. This completes the proof of part (i.

(ii) It follows from (2.6) that if $x \in W_{p}^{2}, \ell=\ell_{x}$ and $M_{n m}=M_{n m}(\alpha, p)$, then

$$
|f(x)|=\left|\int_{1}^{\infty} \int_{1}^{\infty} x(t, r) \alpha(t, r) d r d t+a \ell\right| \leq\|x\|_{2} 2^{\frac{2}{p}} \sum_{n=0}^{\infty} \sum_{m=0}^{\infty} M_{n m}+|a \ell| .
$$

Further, by Minkowski's inequality

$$
\left(1-\frac{1}{T R}\right)^{\frac{1}{p}}|\ell| \leq\left(\frac{1}{T R} \int_{1}^{T} \int_{1}^{R}|x(t, r)-\ell|^{p} d r d t\right)^{\frac{1}{p}}+\left(\frac{1}{T R} \int_{1}^{T} \int_{1}^{R}|x(t, r)|^{p} d r d t\right)^{\frac{1}{p}}
$$

and the first term on the right hand side is $o(1)$. Hence $|\ell| \leq\|x\|_{2}$ and consequently, by (2.7),

$$
|f(x)| \leq\|x\|_{2}\left(|a|+2^{\frac{2}{p}} \sum_{n=0}^{\infty} \sum_{m=0}^{\infty} M_{n m}\right)
$$


for every $x \in W_{p}^{2}$. The additive and homogenous functional $f$ defined by (2.1) is therefore also continuous on $W_{p}^{2}$ and

$$
|f(x)| \leq|a|+2^{\frac{2}{p}} \sum_{n=0}^{\infty} \sum_{m=0}^{\infty} M_{n m} .
$$

Finally, by (2.6), the integral in (2.1) is absolutely convergent. Thus the proof is completed.

Theorem 2.2. $\quad$ (i) If $f$ is a linear functional on $w_{p}^{2}$, then there is a real number a and a real double sequence $\alpha=\left\{\alpha_{n m}\right\}$ such that

$$
f(x)=a \ell+\sum_{n=1}^{\infty} \sum_{m=1}^{\infty} \alpha_{n m} x_{n m}
$$

for every $x=\left\{x_{n m}\right\} \in w_{p}^{2}$ and

$$
\sum_{n=0}^{\infty} \sum_{m=0}^{\infty} m_{n m}(\alpha, p)<\infty
$$

(ii) If $a$ is a real number and $\alpha=\left\{\alpha_{n m}\right\}$ is a real double sequence satisfying (2.9), then (2.8) defines a linear function on $w_{p}^{2}$ with

$$
\|f\|_{2} \leq|a|+2^{\frac{2}{p}} \sum_{n=0}^{\infty} \sum_{m=0}^{\infty} m_{n m}(\alpha, p)
$$

and the series in (2.8) is absolutely convergent for every $x=\left\{x_{n m}\right\} \in w_{p}^{2}$.

Proof. Given any real double sequence $x=\left\{x_{n m}\right\}$, define a bivariate function $x^{*}$ by

$$
x^{*}(t, r)=x_{n m} \text { for } n<t \leq n+1 ; m<r \leq m+1, n=1,2,3, \ldots, m=1,2,3, \ldots
$$

It is easily verified that this defines a one to one correspondence between $w_{p}^{2}$ and a linear subspace $\left(W_{p}^{2}\right)^{*}$ of $W_{p}^{2}$ such that

$$
\ell_{x^{*}}=\ell_{x} \text { and }\left\|x^{*}\right\|_{2} \leq\|x\|_{2} \leq 2^{\frac{2}{p}}\left\|x^{*}\right\|_{2}
$$

Hence given a linear functional on $W_{p}^{2}$, the functional $f^{*}$ defined by

$$
f^{*}\left(x^{*}\right)=f(x)
$$

is linear on $\left(W_{p}^{2}\right)^{*}$. Consequently, by the Hahn-Banach theorem and Theorem2.1, there is a real number $a$ and a real valued bivariate function $\alpha^{*}$, integrable over $\left.(1, \infty) \times 1, \infty\right)$, such that

$$
\sum_{n=0}^{\infty} \sum_{m=0}^{\infty} M_{n m}\left(\alpha^{*}, p\right)<\infty
$$

and, for every $x \in w_{p}^{2}$,

$$
f(x)=f^{*}\left(x^{*}\right)=a \ell_{x^{*}}+\int_{1}^{\infty} \int_{1}^{\infty} \alpha^{*}(t, r) x^{*}(t, r) d r d t=a \ell_{x}+\sum_{n=1}^{\infty} \sum_{m=1}^{\infty} \alpha_{n m} x_{n m}
$$


where $\alpha_{n m}=\int_{n}^{n+1} \int_{m}^{m+1} \alpha^{*}(t, r) d r d t$. Furthermore, for $\alpha=\left\{\alpha_{n m}\right\}$,

$$
\sum_{n=0}^{\infty} \sum_{m=0}^{\infty} m_{n m}(\alpha, p) \leq \sum_{n=0}^{\infty} \sum_{m=0}^{\infty} M_{n m}\left(\alpha^{*}, p\right)
$$

and this completes the proof of (i).

(ii) If $x=\left\{x_{n m}\right\} \in w_{p}^{2} m_{n m}=m_{n m}(\alpha, p)$ and $\ell=\ell_{x}$ then by Hölder's and Minkowski's inequalities, as in the proof of (ii) of Theorem2.1,

$$
\begin{gathered}
f(x)=a \ell+\sum_{n=1}^{\infty} \sum_{m=1}^{\infty} \alpha_{n m} x_{n m} \leq|a \ell|+\sum_{n=1}^{\infty} \sum_{m=1}^{\infty}\left|\alpha_{n m} x_{n m}\right| \\
\leq|a \ell|+2^{\frac{2}{p}}\|x\|_{2} \sum_{n=0}^{\infty} \sum_{m=0}^{\infty} m_{n m} \leq\|x\|_{2}\left(|a|+2^{\frac{2}{p}} \sum_{n=0}^{\infty} \sum_{m=0}^{\infty} m_{n m}\right) .
\end{gathered}
$$

The functional $f$ defined by $(2.8)$ is therefore linear on $w_{p}^{2}$,

$$
\|f\|_{2} \leq|a|+2^{\frac{2}{p}} \sum_{n=0}^{\infty} \sum_{m=0}^{\infty} m_{n m}
$$

and the series in (2.8) absolutely convergent. This completes the proof.

\section{REFERENCES}

[1] S. Banach, Theorie des operations lineaires, Warsaw, 1932.

[2] D. Borwein, Linear functionals connected with strong Cesaro Summability, J. London Math. Soc. 40 (1965), 628-634.

[3] T. J. I. A. Bromwich, An introduction to the theory of infinite series, second ed., Cambridge, 1926 (repr. Macmillan, London, 1955).

[4] G. H. Hardy, On the convergence of certain multiplie series, Proc. Cambridge Philos. Soc. 19 (1916-1919), 86-95.

[5] F. Moricz, Tauberian theorems for Cesaro summable double sequences, Studia Math. 110 (1994), 83-96.

[6] F. Moricz, On the convergence in a restricted sense of multiple series, Anal. Math. 5(1979), 135-147.

[7] F. Moricz, Some remarks on the notion of regular convergence of multiple series, Acta Math. Hungar. 41 (1983), $161-168$.

[8] F. Moricz, Extensions of the spaces c and $c_{0}$ from single to double sequences, Acta Math. Hungar. 57 (1991), $129-136$.

[9] Mursaleen and Osama H. H. Edely Statistical convergence of double sequences, J. Math. Anal. Appl. 288 (2003), $223-231$.

[10] R. F. Patterson, Double Sequence Core Theorems, Internat. J. Math. and Math. Sci. 22 (1999), 785-793.

[11] A. Pringsheim, Zur theorie der zweifach unendlichen Zahlenfolgen, Math. Ann. 53 (1900), 289-321.

[12] A. Pringsheim, Elementare Theorie der unendlichen Doppelreihen, Sitzung-Berichte. der math.-phys. Classe der Akad. der Wissenschafften zu Munchen 27 (1897), 101-152. 